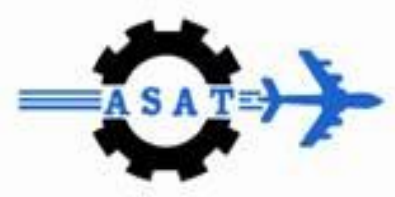

\title{
Design and Implementation of a Novel Planer UWB Monopole Antenna for Multipath Environments
}

\author{
M. Y. Alhefnawy ${ }^{*}$, Aladdin Assisi ${ }^{* *}$, Hosny Almotaafy ${ }^{* * *}$, A. Safwat ${ }^{\dagger}$ and M.I. Youssef ${ }^{\dagger}$
}

\begin{abstract}
In this paper, a new planer monopole ultra-wideband (UWB) antenna is proposed for multipath applications. The proposed antenna is designed to operate from 3.1 to $10.6 \mathrm{GHz}$. It consists of an octagonal shape patch with transmission line edge feed and underlying ground plane. Details of the proposed antenna design and measured results are presented and discussed below. Also a comparison study between the performance of the proposed antenna on FR4 and Taconic TLY-5 materials is carried out.
\end{abstract}

\section{Introduction}

As wireless communication applications require more and more bandwidth, the demand for wideband antennas increases. For instance, ultra wideband radio (UWB) will utilize the frequency-band of 3.1-10.6 GHz. Microstrip patch antennas have the advantages of low cost and compatibility with printed circuit board (PCB) technology, making it possible to integrate the antenna with the RF circuits on the same board. This allows a compact module design. However, a microstrip patch antenna has a limited bandwidth. Bandwidth is limited since the substrate height is limited [1]. To overcome this drawback, the ground plane was truncated and the patch became a planer monopole with underlying truncated ground. The design was optimized using electromagnetic simulation with the Zeland IE3D software. The design goal was to maximize the bandwidth. The proposed antenna covers more than the bandwidth allocated for UWB wireless communications $(3.1 \mathrm{GHz}-10.6 \mathrm{GHz})$. In addition, this design has a planar profile and can easily be integrated in small mobile phones, hand held wireless units, and various remote sensing devices.

The main reason for choosing Zeland IE3D is that it is based on MOM (method of moments) which suits very well with planner structures. We have designed and implemented the proposed antenna on both FR4 and the Taconic TLY-5 materials. FR4 is cheap, while the Taconic material has a low loss tangent. The proposed antenna was successfully implemented on both materials and the simulated results show reasonable agreement with the measured results. In this design, a 3.1 to $10.6 \mathrm{GHz}$ frequency range was intended with $\mathrm{S} 11<-10 \mathrm{~dB}$ and VSWR<1.9. Finally, the performance of the two antennas based on FR4 and TLY-5 has been compared.

\footnotetext{
*AlAzhar University, mohamedyme@gmail.com

***MTC, sisi@msa.edu.eg

**** Electrical Engineering Department. HTI,

${ }^{\dagger}$ Electrical Engineering Department, AlAzhar University
} 


\section{Antenna Design}

The geometry of the proposed antenna is shown in Figure 1 and Figure 2 for FR4 and TLY-5 Materials, respectively. As shown the figures, the antenna consists of an octagonal shape patch with transmission line edge feed and underlying ground plane.

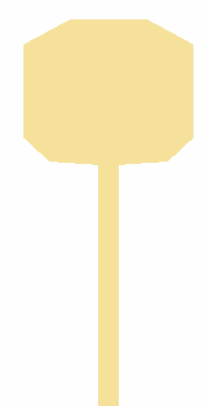

(a)

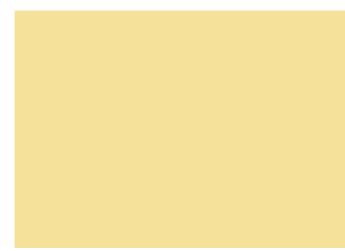

(b)

Figure 1 Geometry of the FR4 proposed UWB antenna: (a) frontView (b) back view

The FR4 antenna was designed and fabricated 0n a $1.6 \mathrm{~mm}$ thickness FR4 substrate with an overall size of $\mathrm{X}$ by $\mathrm{Y} \mathrm{mm}$. The dielectric constant is 4.4 and the tangent loss is 0.02 .

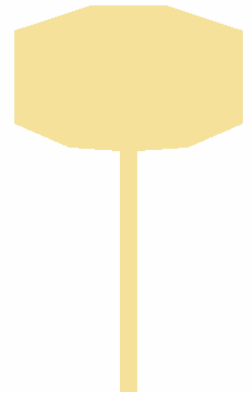

(a)

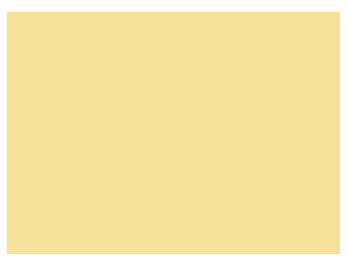

(b)

Figure 2 Geometry of the TLY-5 proposed UWB antenna:

(a) front View (b) back view

\section{Design Approach}

The TLY-5 antenna was designed and fabricated on a $0.78 \mathrm{~mm}$ thick TLY-5 substrate with an overall size less than $35 \mathrm{~mm}$ by $25 \mathrm{~mm}$. The dielectric constant is 2.2 and the tangent loss is 0.002. The idea of this design approach depends on the following three main Transmission Line principles:-

\subsection{Increasing the bandwidth of the antenna by decreasing the Quality factor $(Q)$}

Decreasing the quality factor of the microstrip antenna is an effective way to increase the antenna's impedance bandwidth [2]. Quality factor depends on the reflection coefficient. A lower Quality factor means lower reflection back into the "neck" of the UWB monopole towards the source. When you make the radiating element very wide, the Q factor at the lowest operating frequency becomes low. 


\subsection{Smooth Impedance Transition (No Step Impedance)}

Wherever there is an impedance transition, it should be smooth (i.e. it should change gradually with distance along the wave propagation path). The point where the patch starts and the angle between the lower edges and the ground plane are very important, as here the wave should not experience step impedance transition. There must be a gradual change from the microstrip transmission line impedance to the imaginary characteristic impedance of the patch.

\subsection{A large part of the current wave flows along the patch edges instead of its middle}

When the current wave in the feed line approaches the entrance of the patch, it splits. Large part of the current wave will flow along the edges (with certain angle $\theta$ ). We observed this during the simulation where we manually changed the meshing at the edges, close to the underlying ground plane, to help the simulator. This current wave will experience increasing characteristic impedance when traveling sideways, as the distance between the patch edge and ground plane increases. Characteristic impedance of the patch edges increases as the distance between the patch edges and the underlying ground plane increases.

\section{Electromagnetic Simulation}

The two proposed antennas were simulated and optimized using the Zeland IE3D simulation software. Figure 3 shows the simulated VSWR of the FR4 antenna. It covers the whole UWB Band with VSWR $<1.9$ and the best value $\approx 1.2$ at $6 \mathrm{GHz}$. Figure 4 shows the Simulated S11 of the same antenna. Its return loss is better than $-10 \mathrm{~dB}$ in the whole UWB Band and the best value is better than $-20 \mathrm{~dB}$. Figure 5 shows the Simulated VSWR of the TLY-5 proposed antenna. It covers more than the UWB Band with VSWR $<1.6$ and the best value $\approx 1.1$ at $8 \mathrm{GHz}$. Figure 6 shows the Simulated return loss of the TLY-5 proposed antenna, with S11 $<-13 \mathrm{~dB}$ in the whole UWB Band and best value exceeding $-24 \mathrm{~dB}$.

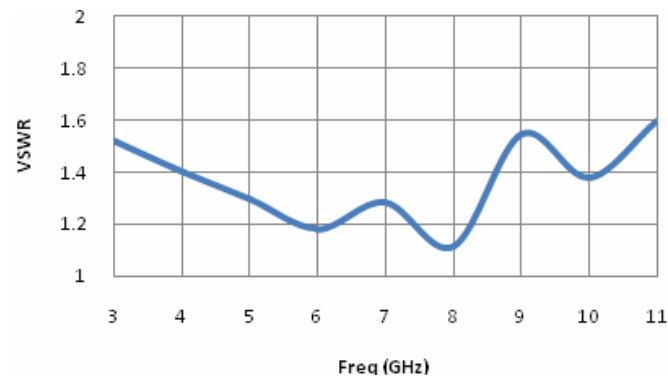

Figure 3 Simulated FR4 antenna VSWR

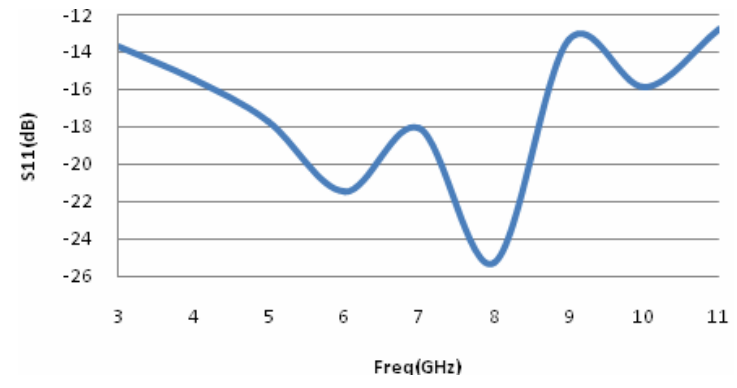

Figure 4 Simulated S11 of the FR4 Antenna 


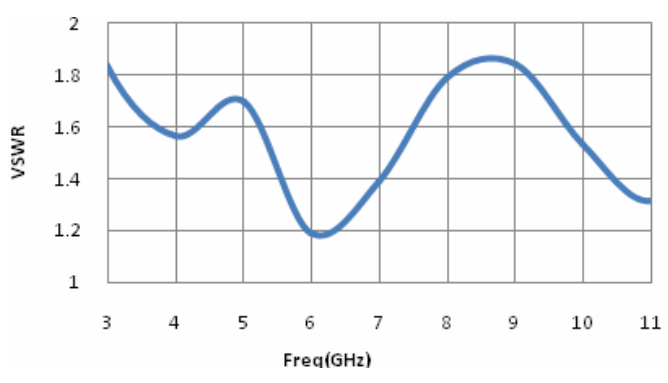

Figure 5. Simulated TLY-5 antenna VSWR

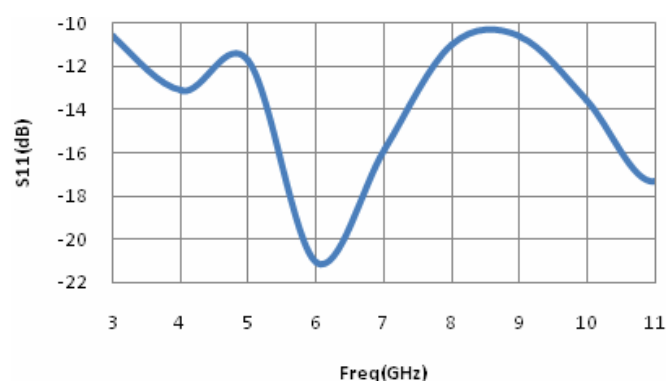

Figure 6 Simulated S11 of the TLY -5 Antenna

\section{Implementation and Measurement}

To validate the proposed design approach, antenna prototypes were designed, fabricated on both FR4 and TLY-5 materials and tested. The photographs of the two fabricated antennas, together with their measured return losses vs. frequency are shown in Figures 7 and 8 , respectively.

If we consider $\mathrm{S} 11 \leq-10 \mathrm{~dB}$ a limiting value for acceptable matching, it can be evidently seen that the TLY-5 antenna achieves an ultra-wide bandwidth starting from $2.23716 \mathrm{GHz}$ and ending at $15.4495 \mathrm{GHz}$. The center of this band is $8.84333 \mathrm{GHz}$ and the ratio

$$
\frac{B W}{f_{c}}=\frac{2\left(f_{\max }-f_{\min }\right)}{f_{\text {max }}+f_{\text {min }}}=\frac{2(15.4495-2.23715)}{15.4495+2.23715}=1.494
$$

From these results, it can be concluded that a bandwidth of $149.4 \%$ has been achieved with the TLY-5 antenna. A good agreement is evident between experimental results and simulated ones. For the FR4 antenna the bandwidth starts at $2.18942 \mathrm{GHz}$ and ends at $11.84 \mathrm{GHz}$. Applying the above mentioned BW definition it can be seen that the FR4 antenna has a $137.6 \%$ bandwidth.

\section{Conclusion}

In this paper, we introduce a new approach for designing a novel octagonal planer UWB monopole antenna for ultra wideband multipath environments applications. This approach enhances the most important antenna parameters for indoor application VSWR and Return Loss. Upon this approach, two antennas with two differences substrate materials (FR4, TLY-5) are presented and their results are given. The measured bandwidth of both antennas exceeds 137\% which has not been previously achieved by any PCB antenna. 


\section{References}

[1]. M Karlsson, S Gong, Wideband patch antenna array for multi-band UWB, , C Norrkoping Proc. of IEEE 11th SCVT, 2004 - itn.liu.se.

[2]. Kin-Lu Wong, Compact and Broadband Microstrip Antennas, Wiley, 2002, pp. 13.

[3]. J. Ollikainen and P. Vainikainen, "Design And Bandwidth Optimization Of Dual-Resonant Patch Antennas", Helsinki University of Technology Radio Laboratory Publications, Espoo, March, 2002.

[4]. Pekka M. T. Ikonen, , Pekka Alitalo, Sergei A. Tretyakov, Pekka M. T. Ikonen, Pekka Alitalo, Sergei A. Tretyakov, "On Impedance Bandwidth of Resonant Patch Antennas Implemented Using Structures with Engineered Dispersion”, IEEE, Nov 2002.

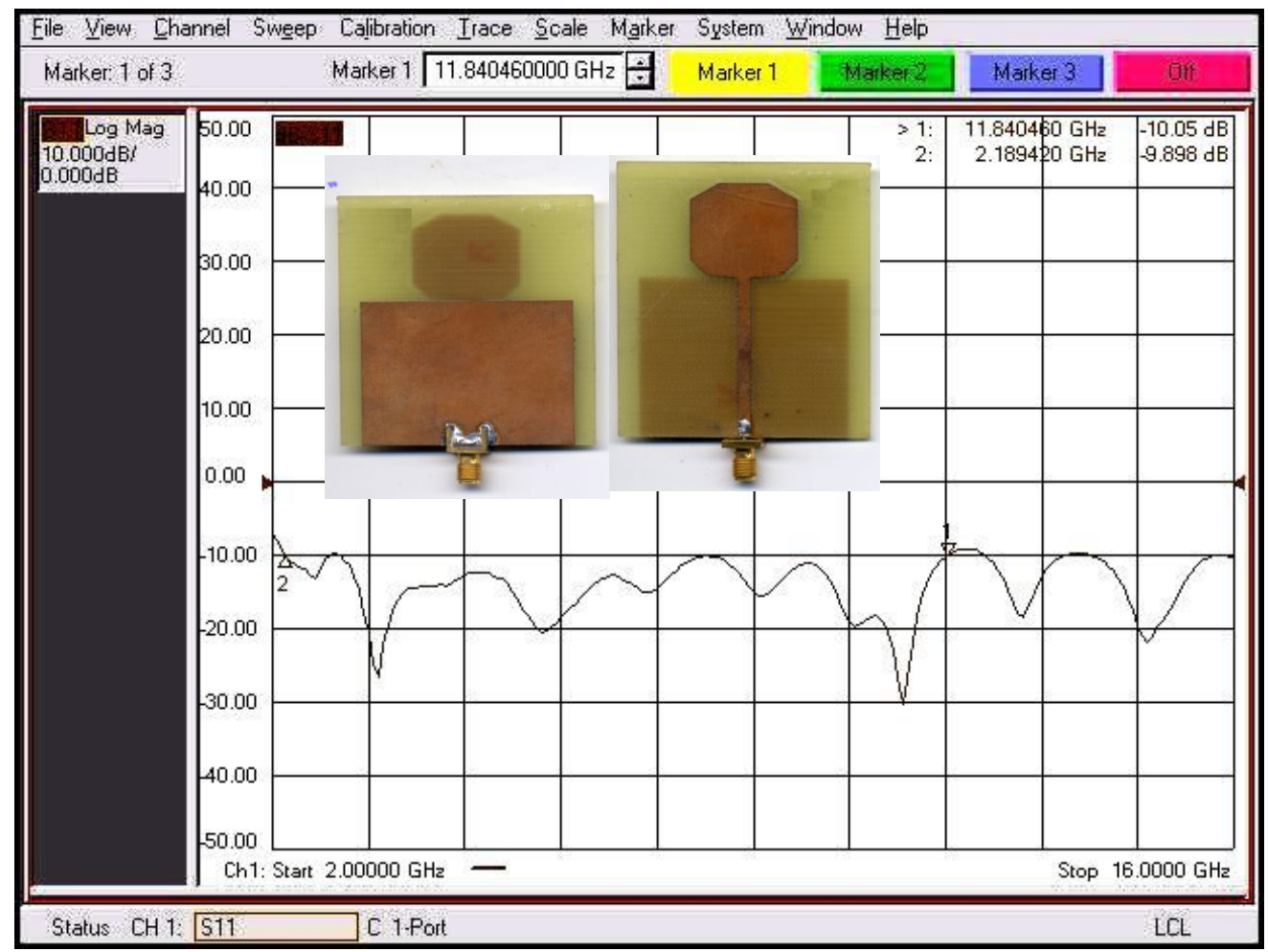

Figure 7. Photographs and Measured returned loss of the FR4 implemented Antenna 


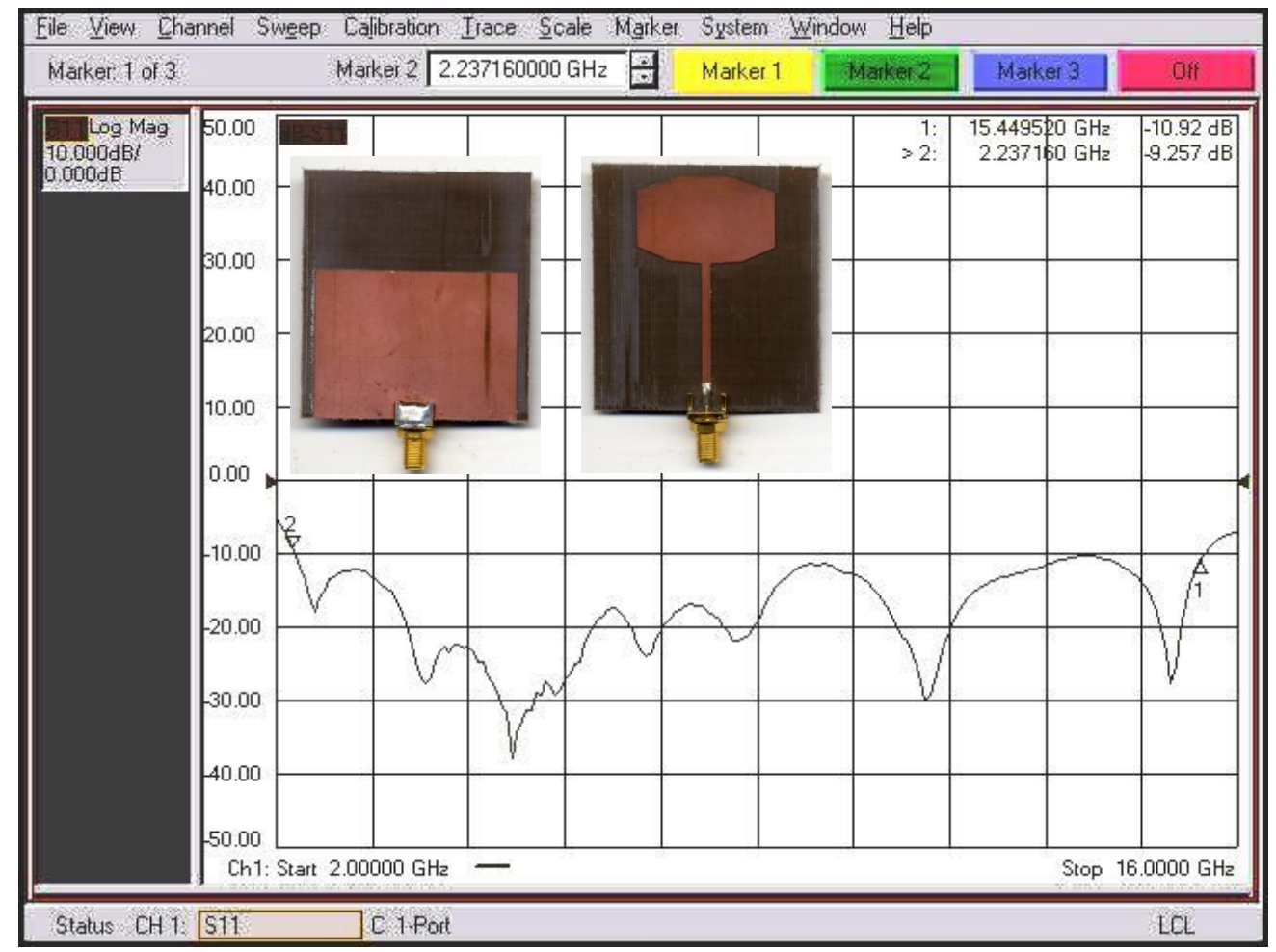

Figure 8. Photographs and Measured returned loss of the TLY-5 implemented Antenna 